\title{
Imperio, Estado y Nación en las relaciones entre chilenos y británicos durante el proceso de independencia hispanoamericano, 1806-1831
}

\author{
Andrés Baeza Ruz ${ }^{1}$ \\ Recibido: 10 de abril de 2017 - Aceptado: 15 de mayo de 2017
}

\begin{abstract}
Resumen
El proceso de independencia chileno e hispanoamericano transcurrió en medio de un complejo escenario de rivalidades entre los principales imperios europeos, como Gran Bretaña, Francia y España. Algunos autores sostienen que los Estados hispanoamericanos fueron el resultado de esas luchas, lo que nos indica que para comprender a cabalidad el proceso de independencia es necesario considerar este escenario geopolítico. En este estudio nuestro objetivo es situar el proceso de independencia chileno en dicho escenario, con un particular foco en las dinámicas y cambiantes relaciones experimentadas entre chilenos y británicos durante el periodo. Sostenemos que dichas relaciones fueron fundamentales para catalizar procesos de reflexión y discusión acerca de lo que era o debía ser la nación chilena. Para desarrollar el argumento, el trabajo comienza por analizar el escenario geopolítico, para luego profundizar en la naturaleza de las relaciones británico-chilenas y, finalmente, identificar tres etapas de dichas interacciones, determinadas por el rol cambiante de Gran Bretaña en el escenario internacional.
\end{abstract}

Palabras clave: independencia, imperialismo, reconocimiento, Chile, Gran Bretaña

Empire, State and Nation in the relationships between Chilean and Britons people during the process of Spanish-American independence, 1806-1831

\footnotetext{
Abstract

The process of Chilean and Spanish American independence took place in a complex scenario of rivalries among the major European Empires, such as
}

1 Chileno. Doctor en Historia de América Latina, Universidad de Bristol, Reino Unido. Investigador Postdoctoral, Proyecto Fondecyt Postdoctorado 3170966, Instituto de Historia, Pontificia Universidad Católica de Chile. E-mail: afbaeza@gmail.com 
Britain, France and Spain. Some authors argue that the Spanish-American states were the result of these struggles, which indicates that in order to fully understand the process of independence it is necessary to consider this geopolitical scenario. The objective of this study is to situate the process of Chilean independence in this scenario, with a particular focus on the dynamics and changing relations experienced between Chilean and British people during that period. We believe that these relations were fundamental to catalyze processes of reflection and discussion about what was or should be the Chilean nation. In order to develop the argument, the work begins by analyzing the geopolitical scenario, then deepening the nature of British-Chilean relations, and finally identifying three stages of such interactions, determined by the changing role of Great Britain in the international arena.

Keywords: Independence, imperialism, reconnaissance, Chile, Great Britain

\title{
Império, Estado e Nação nas relações entre chilenos e britânicos durante o processo de independência hispano-americana, 1806-1831
}

\begin{abstract}
Resumo
0 processo de independência do Chile e da América Latina aconteceu em meio de um complexo cenário de rivalidades entre os principais impérios europeus, como a Grã-Bretanha, França e Espanha. Alguns autores argumentam que os estados latino-americanos foram o resultado dessas lutas, o qual indica que para compreender completamente o processo de independência é necessário considerar este cenário geopolítico. Neste estudo o nosso objetivo é situar o processo de independência do Chile neste cenário, com um foco especial nas dinâmicas e cambiantes relações experimentadas entre chilenos e britânicos durante o período. Argumentamos que ditas relações foram fundamentais para catalisar processos de reflexão e discussão sobre o que era ou deveria ser a nação chilena. Para desenvolver o argumento, 0 trabalho começa por analisar o cenário geopolítico, para logo aprofundar na natureza das relações britânico-chilenos e, finalmente, identificar três estágios de tais interações, determinadas pela mudança do papel de GrãBretanha no cenário internacional.
\end{abstract}

Palavras-chave: independência, imperialismo, reconhecimento, Chile, Grã-Bretanha

Las relaciones entre Chile y Gran Bretaña tienen una larga data. Tempranamente, como ha destacado William Edmundson, una vez establecido el dominio español sobre el territorio, la presencia de piratas, corsarios, viajeros y contrabandistas se hizo cada vez más recurrente en las costas chilenas (Edmundson, 2009: 7-26). Dicha presencia obedecía también a ciertas lógicas: la creciente rivalidad imperial entre imperios como Gran Bretaña, España y Francia, y la utilización de medios de diversa índole 
para minar el poderío de su rival. Si bien esto es evidente para el periodo colonial, no lo es tanto para años posteriores. Autores como John Mayo (2007) han abordado estas relaciones a partir de la década de 1850, en que las inversiones británicas en Chile, así como su participación en diversas actividades económicas, se presentaban como argumento suficiente para catalogar la relación como "imperial". En esa lógica, la incipiente presencia británica durante los años de la independencia sería considerada como un antecedente fundante de dicha relación. En nuestro caso, creemos, por el contrario, que la independencia es un proceso con lógicas propias y que no necesariamente pueden trazarse líneas de continuidad hacia el futuro. De hecho, el proceso iniciado en 1808, tras el aprisionamiento del rey Fernando VII, se caracterizó por su dinámica cambiante y sus ritmos diversos, que hacían impredecible en 1810, lo que iba a transcurrir en 1818. Lo mismo puede decirse de la relación entre Chile y Gran Bretaña. Como veremos, la manera en que los chilenos ${ }^{2}$ percibían y representaban al imperio británico, cambió de acuerdo con las circunstancias y no siempre primó el carácter "imperialista" de la relación. Por ello se hace necesario detenerse en algunos procesos de la independencia que pueden dar luces acerca de cómo estas interrelaciones influenciaron el proceso mismo de independencia, como el ulterior proceso de construcción del Estado-nación.

En ese sentido, es necesario identificar también cómo evolucionó la política británica hacia Chile a lo largo del periodo de independencia y las consecuencias que esto conllevó para los chilenos en sus modos de identificar la relación con dicho imperio. Sostenemos que los diversos modos en que dicha política fue vivenciada por los propios chilenos tendrán importantes consecuencias para la configuración de lo que podría llamarse una "identidad" nacional o protonacional y la representación del lugar de Chile en América y el mundo. En términos generales, el curso de las guerras napoleónicas en Europa y el resultado del proceso de independencia, es decir, el surgimiento de nuevos Estados en la América española, determinaron el modo en que los políticos británicos imaginaron su acercamiento con Chile. Por otra parte, las cambiantes alianzas y relaciones entre las potencias europeas durante las guerras napoleónicas determinaron, a su vez, el modo en que los chilenos percibieron y representaron el papel de Gran Bretaña y de los británicos, pasando de ser un enemigo a un aliado y más tarde, un modelo a seguir.

2 Utilizamos el término "chileno" en un sentido práctico, refiriéndonos a los habitantes del territorio y no a quienes se identifican con una determinada nacionalidad, pues asumimos que en el periodo abordado en este estudio la identidad chilena recién comenzaba su proceso de construcción. 


\section{La reconfiguración de las alianzas inter-imperiales y la independencia de Chile}

El proceso de independencia en Chile e Hispanoamérica se desarrolló en un contexto geopolítico de rivalidades inter-imperiales europeas que involucraban fundamentalmente a España, Francia y Gran Bretaña. Los resultados de las guerras napoleónicas en Europa determinaron drásticos cambios en las alianzas entre dichos imperios. El 2 de mayo de 1808, día en que Napoleón ingresó a España al mando de aproximadamente veinte mil hombres, fue un punto de inflexión que marcó una reconfiguración estratégica de las alianzas entre dichos imperios. Desde ese momento, y sobre todo a raíz del cautiverio de Fernando VII en Bayona y la entronización de José Bonaparte como rey de España, el antiguo pacto de familia firmado entre los Borbones de Francia y España, que mantenía a las coronas de ambos Estados como aliados frente a Gran Bretaña, se disolvió (Elliot, 2006). Esto trajo como consecuencia la conformación de una nueva alianza entre España y Gran Bretaña con el objetivo de derrotar a las fuerzas napoleónicas y expulsarlas de la Península Ibérica. Este fue el escenario que sorprendió a los hispanoamericanos al conocer las noticias del aprisionamiento del rey Fernando VII. Un escenario radicalmente diferente al que tuvieron que enfrentar en 18061807, cuando un contingente de aproximadamente mil setecientos soldados británicos invadió y ocupó la ciudad de Buenos Aires y luego Montevideo. En dicha oportunidad, no hubo dudas respecto a la necesidad de expulsar al enemigo invasor de los dominios del rey y la lealtad a Fernando VII se expresó sin matices (Gallo, 2001). Para el caso chileno, dicho acontecimiento revistió una serie de consecuencias, siendo particularmente importante la movilización de la sociedad a fin de implementar un plan de defensa del territorio frente a una posible invasión británica (Ossa, 2010: 442-446). De hecho, en mayo de 1808, cuando se produjo la invasión napoleónica al territorio español, todavía se discutía en Santiago la posibilidad de enviar contingentes militares a Buenos Aires a fin de defender la ciudad de una nueva expedición británica de siete mil hombres que se había organizado en Irlanda, en la isla de Cork, al mando de Arthur Wellesley, el Duque de Wellington (D'Arjuzon, 2003: 118-119).

Para los hispanoamericanos, ignorantes de lo que acontecía en la Península Ibérica debido al desfase en la recepción de la información, Gran Bretaña todavía era visualizada como el principal enemigo en este juego de alianzas. Solo en septiembre de 1808, es decir, cuatro meses después de las abdicaciones de Bayona, llegaron a Santiago las noticias de lo acontecido en España, obligando a las autoridades criollas a cambiar tanto de enemigo como de estrategia en los preparativos de la defensa del territorio (Barros Arana, 1854: 125). Al menos hasta 1810, el posible enemigo invasor pasaría a ser Francia, mientras que la percepción hacia Gran Bretaña mutaría a 
la de un poderoso aliado en un contexto de temor e incertidumbre. Como han demostrado recientes estudios, la formación de la Junta gubernativa en septiembre de 1810 en Santiago, se convirtió en un nuevo punto de inflexión, pues el virrey del Perú Fernando de Abascal, lejos de aceptar las medidas políticas que comenzaron a tomarse en Chile, invadió el territorio a fin de mantener el control en ausencia del rey (Ossa, 2016). Para los líderes del proceso político iniciado en 1810 en Santiago, esto sería interpretado como una invasión ilegítima y nuevamente cambiaría el enemigo a vencer, esta vez, encarnado en la figura de Abascal. Por su parte, Gran Bretaña, lejos de involucrarse en forma activa en el conflicto, daría inicio a su política de neutralidad, aunque siempre actuando solapadamente en favor de los americanos (Waddell, 1987).

Sin este trasfondo geopolítico resulta difícil comprender el derrotero de las revoluciones de independencia hispanoamericanas, que también se vieron condicionadas por el curso de las guerras napoleónicas y por la reconfiguración de las relaciones entre los imperios europeos. De hecho, Annick Lamperiere ha señalado que este contexto es tan crucial que los nuevos Estados hispanoamericanos fueron "el producto final e incluso los protagonistas de una larga lucha inter-imperial" (Lamperiere, 2013: 18). La referencia al protagonismo de los nacientes Estados hispanoamericanos no es menor, por cuanto el proceso de independencia tuvo un significado político mayor, que afectaría radicalmente a la configuración del poder entre los imperios europeos. Para Ralph Blaufarb, el conflicto de la independencia, enmarcado en este escenario, estableció el dilema de la "cuestión occidental", en el sentido de que "no se trataba tanto de la independencia per se, sino que de cómo iba a afectar al orden geopolítico". Y en ese dilema, el rol de Gran Bretaña resultaba crítico, por cuanto todos los otros poderes como España, Francia, Portugal y Estados Unidos temían que alcanzara la "hegemonía global" por medio de la "transformación de Sudamérica en una segunda Indostán", en relación con el rol de la Compañía de las Indias Orientales (Blaufarb, 2007: 747). En consecuencia, frente a un Napoleón derrotado y un imperio español en proceso de desintegración, con la independencia de Hispanoamérica estaba en juego el reordenamiento geopolítico en Europa en favor de la hegemonía británica.

\section{¿Expansión de intereses británicos o política imperialista?}

Considerando lo planteado en la sección anterior, resultaba plausible que Gran Bretaña actuase en favor de los hispanoamericanos a fin de debilitar a España y de ese modo asegurar su hegemonía. No obstante, el involucramiento de Gran Bretaña en el conflicto entre el monarca español y los 
hispanoamericanos fue ambigua y cambiante y obedeció a diversos intereses, tanto particulares como estatales, que no siempre eran coincidentes. Desde un punto de vista estatal, la política de neutralidad se mantuvo vigente durante todo el periodo, pese a lo cual los líderes hispanoamericanos buscaron incesantemente el apoyo económico, logístico y político de Gran Bretaña para afrontar el conflicto. Ya en 1811, prácticamente todos los gobiernos provisorios establecidos enviaron representantes a Londres a negociar el apoyo oficial de la corona británica y de otros Estados como Francia a la insurgencia (Racine, 2010). En el caso chileno, Francisco Antonio Pinto fue enviado a Londres en diciembre de 1813 por el Congreso Nacional con instrucciones que señalaban claramente la naturaleza de su misión: "viajar a las cortes de Roma, Francia e Inglaterra" a fin de ganar el reconocimiento para la causa. Las Instrucciones especificaban, además, todo aquello que Pinto debía ofrecer a cambio como parte de la negociación: libre comercio, tierras, beneficios tributarios y la formación de una "Confederación sudamericana" que diera garantías de estabilidad (Montaner Bello, 1961: 441-446). No obstante, los resultados de dichas negociaciones fueron infructuosos, puesto que no logró persuadir a Lord Castlereagh de ceder en su política de neutralidad. En ese sentido, es posible afirmar que, a nivel estatal, el interés de Gran Bretaña respecto a la independencia chilena era prácticamente nulo. Pese a ello, las Instrucciones reflejan que ya hacia 1813 existía una idea de que era necesario construir una imagen de Chile como un destino atractivo, muy diferente a lo obrado por Luis Muñoz de Guzmán, que había optado por resaltar lo contrario a fin de persuadir a posibles invasores.

Distinto era el rol de los actores privados, especialmente de los mercaderes y comerciantes. Sus intereses comerciales se habían hecho notar incluso antes de 1808 y, como apuntamos más arriba, hay quienes señalan que fueron los mercaderes británicos quienes estuvieron tras el diseño de diversos planes para invadir Chile durante el periodo colonial. Durante el proceso iniciado en 1810, aprovecharon tempranamente la apertura comercial decretada por la Junta, como lo demuestra el caso de John James Barnard, quien llegó a Valparaíso en 1811. Desde diversas corrientes se ha interpretado esta expansión de intereses comerciales como la expresión de una política imperialista de Gran Bretaña hacia Chile, que buscaba mantener a este último en una posición de subordinación y dependencia (Mayo, 1981; Salazar, 2011). No obstante, como hemos señalado, a nivel "oficial" no existía una política explícita destinada a extender el dominio imperial hacia Chile, con la excepción del Plan para invadir Chile entregado a Robert Craufurd en octubre de 1806, con posterioridad a las invasiones de Buenos Aires. La pregunta que cabe hacerse, entonces, es si cabe hablar de imperialismo cuando no existe una política "oficial" o bien, si cualquier presencia de actores privados en territorios fuera de los contornos del imperio "formal" pueden considerarse también como una expresión de dominio imperial. 
Si aplicamos la tesis de Peter Marshall se puede afirmar que la extensa presencia británica en Hispanoamérica incluso antes de 1808 se trataba más bien de una etapa de "expansión" de intereses, en el cual se involucraron actores privados, sin que necesariamente deba hablarse de imperialismo. Gobierno y coerción, a juicio de Marshall, son dos elementos fundamentales para definir las relaciones imperiales (Marshall, 2005: 13). En este caso, no es claro que estuviesen presentes, al menos si se considera la evolución de los acontecimientos entre 1808 y 1830, con la sola excepción de las invasiones de 1806-1807, que fue el único momento en el cual se hizo explícita una política oficial por incorporar a América del Sur al imperio británico. Con posterioridad a dichas invasiones, la política británica hacia Hispanoamérica no consideraría una invasión como una estrategia a seguir, sino que sobre todo una estrategia de presión para la apertura al comercio y la legalización de las actividades de los hasta ese entonces "contrabandistas". Esto último ha sido interpretado como la manifestación de una política de "imperialismo informal", a partir de los postulados de Gallagher y Robinson acerca de la política imperial británica en el siglo XIX, basada fundamentalmente en la expansión comercial sin necesidad de existir coerción y gobierno, como apunta Marshall (Mayo, 1981 y 1987).

En ese sentido, para el periodo abordado en este estudio, calificar la política hacia Hispanoamérica bajo dicho rótulo resulta complejo. En primer lugar, porque no existió una política unívoca y homogénea hacia todos los territorios, sino que fue diferente en función de la importancia relativa que cada uno tenía a ojos de las autoridades británicas. En ese sentido, resulta evidente que había prioridades, como lo muestran las invasiones a Buenos Aires en 1806 y el reconocimiento temprano de la independencia de las Provincias Unidas de la Plata, Colombia y México a fines de 1824, durante la administración de George Canning. Chile, en cambio, ocupaba un lugar algo ambiguo en el plan trazado por autoridades como William Windham, Castlereagh o el mismo Canning. No era un virreinato ni un territorio económicamente relevante como Buenos Aires o México, pero sí ocupaba un lugar estratégico en el mapa que permitía a los británicos conectar el Atlántico y el Pacífico y así establecer un circuito comercial hacia un océano que todavía no dominaban y en el que de a poco se asomaba el creciente poderío de Estados Unidos.

En segundo lugar, desde el punto de vista de los hispanoamericanos en general y chilenos en específico, no es claro que la política británica fuese percibida como imperialista. Si tomamos en cuenta los aportes de autores como Matthew Brown (2006) y Louise H. Guenther (2004) para los casos colombiano y brasilero durante este mismo periodo, es evidente que, para poder caracterizar una relación entre dos partes como imperialista, quienes "sufren" la condición de colonizados deberían percibirla como tal. Aunque 
Brown aporta suficiente evidencia como para demostrar que en el caso colombiano sí existía dicha percepción y sobre todo, el "temor" a perder la soberanía, Guenther no identifica lo mismo para el caso brasilero. En el caso chileno, como mostraremos a continuación, la percepción hacia Gran Bretaña fue cambiante, divergente $y$, a veces, contradictoria, por lo que es difícil establecer, más allá del expansionismo económico, que la relación era imperialista. Esto no significa que el imperialismo en cuanto política haya estado ausente, sino que fue una de varias posibilidades que definieron las relaciones entre Chile y Gran Bretaña entre 1806 y 1830.

\section{El fantasma de una invasión británica y la respuesta de las autoridades coloniales}

En un primer momento (1806-1808), las interacciones entre chilenos y británicos se llevaron a cabo de manera preminente a nivel inter-estatal y se expresaron tanto en los planes para invadir Chile (en cuanto colonia, parte integrante de España) como para defenderlo. En esta fase sí es evidente que la política británica hacia Chile fue definida principalmente por el imperialismo, pues se desarrolló en un momento de apogeo de las pugnas inter-imperiales, que tuvieron su correlato en Hispanoamérica y Chile. Hacia 1806 Europa se encontraba en plena guerra, y Francia y España habían establecido una alianza contra Gran Bretaña. En diciembre de 1805 se había llevado a cabo la batalla de Trafalgar, que aseguró la hegemonía británica en los mares y obligó a Napoleón a replantear su estrategia, activando el bloqueo continental (Robson, 2012). Por otro lado, fue a partir de dicho momento en que los británicos comenzaron a visualizar la posibilidad de una invasión a Hispanoamérica, que se reforzaba con el hecho de que la flota española había sufrido severos daños y difícilmente podrían acudir en ayuda de los americanos. Fue en ese contexto que se llevaron a cabo las invasiones inglesas a Buenos Aires a partir de junio de 1806, lo que gatilló la resistencia de los habitantes de la ciudad y la movilización de sus autoridades, quienes solicitaron ayuda a sus vecinos, entre ellos Chile, a fin de mantener libre a la ciudad del dominio británico (Gallo, 2001). Esto instaló el temor de que una invasión británica a Chile era inminente y que por tanto la sociedad debía movilizarse en defensa del territorio (Barros Arana, 1854).

Dichos temores tenían un sustento razonable y no solo por la presencia británica al otro lado de la Cordillera de los Andes, sino que la intención de invadir Chile había sido estudiada en variadas oportunidades, especialmente por grupos de mercaderes británicos (Barros, 1970). Hubo al menos cuatro planes, entre ellos el controvertido Plan Maitland (1801) para invadir Chile e incorporarlo al imperio formal británico (Terragno, 2001). El triunfo parcial británico en las invasiones a Buenos Aires motivó a las autoridades a poner 
en marcha un nuevo plan, expresado en las "Instrucciones para invadir Chile", que Lord Robert Windham entregó al brigadier general Robert Craufurd en octubre de 1806. La importancia de este plan, más allá de constatar la existencia de una política imperialista hacia Chile en 1806, es que resume la imagen construida acerca de Chile, no como un lugar o mercado en sí mismo, sino que siempre en relación a otros, particularmente a Perú. En otras palabras, aunque el Plan concebía a Chile como un lugar estratégico para expandir las redes comerciales, lo describía como un lugar sin identidad propia y tan solo relevante por conectar otros lugares más importantes. De hecho, la primera carta del Plan señalaba que el objetivo de la invasión sería: "Fomentar la introducción de productos británicos en Perú desde los puertos de Chile", agregando que "dicho intercurso debe brindar oportunidades para imprimir las ventajas de la conexión británica en las mentes de los peruanos y predisponerlos a que concurran a cualquier medida que pueda ser tomada para subvertir el gobierno español". ${ }^{3}$ Dicho de otro modo, el objetivo de ocupar militarmente Chile no era otro que abrir y conectar rutas comerciales hacia otros destinos, siendo Chile poco más que un lugar de tránsito. Curiosamente, esta imagen sería reproducida por las mismas autoridades chilenas una vez ya declarada la independencia, cuando, al momento de persuadir al misionero protestante James Thomson de viajar a Chile a instaurar el sistema lancasteriano de educación, lo hicieron bajo el argumento de que podría estar en Chile unos meses para después ir a Perú (Baeza, 2015: 75).

Desde el lado chileno, en cambio, la posibilidad de una invasión, latente más que nunca una vez que se supo que las tropas británicas habían ocupado Buenos Aires, generó una reacción contraria, más allá de los miedos y temores a ser invadido; esto es, pensar quizás por primera vez en este contexto acerca de Chile como una comunidad con cierta entidad propia y diferenciada de otras como Buenos Aires. A raíz de estas invasiones, el gobernador Luis Muñoz de Guzmán, asesorado por Judas Tadeo Reyes, elaboró un plan de defensa, que puso en evidencia la conciencia acerca de la marginalidad y pobreza del territorio, así como la falta de disciplina de sus milicias. Serán esas características, no obstante, las que se resaltarán en el plan a la hora de intentar desactivar cualquier posibilidad de un ataque. Un batallón de caballería compuesto por fieros guerreros armados por "cuchillos, lanzas y machetes" y la destrucción de las cosechas cercanas a la costa para resaltar la imagen de territorio pobre y así desincentivar cualquier invasión, fueron parte de la estrategia diseñada. ${ }^{4}$ Esto reproducía una antigua estrategia

3 "Instructions to Craufurd", 30 de octubre de 1806, The National Archives at Kew, War Office (WO) 1/161, fs. 136-154.

4 "Informe de Luis Muñoz de Guzmán para defender el reino de los ingleses, en vista del peligro que reviste una invasión luego de la ocupación de Bs. As.", Santiago, 27 de octubre de 1806, Archivo Nacional Histórico de Chile, Fondo Varios, vol. 237, f. 183. 
para protegerse de posibles ataques por mar, que consistía en convencer a los posibles invasores de la pobreza del territorio para así persuadirlos de no desembarcar (Cubitt, 1976: 15).

Frente a la posibilidad de una nueva invasión a Buenos Aires en 1807, y la consecuente petición de ayuda al cabildo de Santiago por parte del virrey Liniers, se activó un debate en torno a la conveniencia de ir en su ayuda. Diversos "cuerpos" como el Tribunal del Consulado, la Universidad de San Felipe, la Real Audiencia, el Cabildo Eclesiástico y el Tribunal de Minería enviaron informes con sus opiniones respecto a lo que debía hacerse, sumado a las propias opiniones del nuevo gobernador, Francisco Antonio García Carrasco, y Judas Tadeo Reyes. Estos informes muestran que no había consenso en torno a lo que debía hacerse, así como tampoco en torno a qué era exactamente aquello que se estaba defendiendo: ¿Era la gran patria americana o esta comunidad próxima de chilenos? ¿Por qué gastar recursos que no se tenían en ir a defender Buenos Aires? La opinión mayoritaria era la de acudir en ayuda de ser necesario. Mientras algunos lo argumentaban desde el lado emocional, arguyendo que eran "todos hijos de una misma madre y miembros de una misma familia", ${ }^{5}$ destacando que hablaban un mismo idioma y profesaban una misma religión, otros lo hacían desde un punto de vista meramente estratégico: enviar unas cuantas tropas para detener al enemigo lejos del territorio propio, asumiendo que, además, la caída de Buenos Aires implicaría la desconexión e incomunicación de Chile con el resto del imperio. García Carrasco, de hecho, fue quien más se aventuró en afirmar que era necesario retener al enemigo lejos, eludiendo las apelaciones de tipo emocional. Además, lo que estaba en juego era la propia supervivencia de esta comunidad, por lo que se argumentó en favor de evitar que el enemigo se aproximarse al territorio chileno por cuanto la magnitud de las consecuencias de una posible invasión podía ser insospechada.

En definitiva, esta primera etapa de interrelaciones británico-chilenas a partir de las invasiones a Buenos Aires, tuvo como un elemento central la existencia de una política explícita por parte de las autoridades británicas de incorporar a Chile a su imperio formal, utilizando dos mecanismos propios de una dominación: coerción (a través de una invasión) y gobierno (reemplazando al monarca español por el británico). Como contrapartida, la política imperial española también se activó, aunque mediante los cauces establecidos; esto es, que el gobernador de Chile hiciera uso de sus facultades, procurando diseñar estrategias adecuadas para la defensa del territorio. Fue mediante la

5 "Judas Tadeo Reyes a la Universidad de San Felipe", Santiago, 4 de julio de 1808, Archivo Nacional Histórico, Fondo Capitanía General, vol. 819, f. 15. En una carta al Cabildo de Santiago, añadió: "somos hijos de un mismo rey", Santiago, 4 de julio de 1808, Archivo Nacional Histórico, Fondo Capitanía General, vol. 819, f.10. 
iniciativa de esta autoridad que también se discutió acerca de qué definía a la comunidad de chilenos que estaban en una situación crítica y determinar si acaso debían velar únicamente por su autodefensa o ir en ayuda de sus vecinos.

\section{Neutralidad británica e intereses particulares}

De 1808 a 1817 las interacciones fueron llevadas a cabo principal, pero no exclusivamente, entre los actores "no estatales", como resultado de la política de neutralidad de Gran Bretaña, que implicó un retroceso por parte de las autoridades británicas en cuanto a definir una política oficial por incorporar a Chile a su imperio formal. Dicho retroceso no impidió a determinados actores privados emprender rumbo hacia Hispanoamérica y Chile en busca de nuevas oportunidades. En el caso de mercaderes y comerciantes se trataba de una situación especial, por cuanto su presencia no era nueva en Chile, pero sí ilegal. El advenimiento de la Junta gubernativa en 1810 fue una oportunidad para legalizar aquello que era ilegal, lo que se concretó mediante la aprobación del Decreto de comercio libre. Con respecto a los militares y marinos, su arribo a Hispanoamérica y Chile, tanto en calidad de mercenarios como de aventureros, se produjo a nivel masivo debido al fin de las guerras napoleónicas y a la necesidad de continuar con sus actividades en un nuevo escenario. Las guerras de independencia fueron, así, una instancia para unirse a un ejército o a una escuadra y de algún modo mantener parte esencial de su vida previa a 1815.

Esta mayor apertura a la presencia británica en Chile se explica también por el nuevo escenario geopolítico iniciado en 1808. Cuando Francia invadió España en mayo de ese año, las relaciones entre las potencias europeas se reconfiguraron, lo que llevó a Gran Bretaña y España a convertirse en aliados. Esto llevó a los chilenos a cambiar sus percepciones y actitudes hacia Gran Bretaña y concentrarse en la defensa de su territorio en nombre de su rey, aunque todavía sospechando de las intenciones de los británicos. De hecho, un poco antes de conocerse la noticia del aprisionamiento de Fernando VII, García Carrasco activó un plan para expulsar a los extranjeros del reino de Chile que no profesaran la religión católica y que no fuesen "útiles" a los intereses del reino (Amunátegui, 1876: 330-335). Esto principalmente apuntaba a los británicos que se encontraban avecindados en el territorio y fue la primera vez en que se fomentó la permanencia de personas que practicasen oficios útiles para el interés común. Dicha práctica se haría extensiva posteriormente, en las instrucciones que el Congreso de Chile despachó a Francisco Antonio Pinto en 1811, cuando fue enviado como emisario a Londres y que luego se repetiría en las misiones de Irisarri y Mariano Egaña. En ambos documentos las autoridades definieron claramente su interés por atraer a territorio chile- 
no a trabajadores y "sabios" europeos que pudiesen aportar con su trabajo en ámbitos claves de la organización política y económica (Montaner Bello, 1961: 441-446). Esto también situaba en el imaginario de las autoridades criollas a Gran Bretaña como un lugar desde el cual podía fomentarse la inmigración de personas idóneas, que pudiesen contribuir al desarrollo de diversas actividades económicas y a la difusión de conocimientos útiles por medio de instituciones de índole educativa. En ese sentido, también comenzó a difundirse la imagen de Chile como un lugar abierto a recibir extranjeros.

En ese sentido, la formación de la Junta en 1810, así como las medidas tendientes a una mayor apertura, impulsaron la llegada de diferentes actores. Como dijimos anteriormente, en un primer momento se trató sobre todo de mercaderes y mercenarios, para luego dar paso a actores diversos como misioneros y educadores. Todos ellos llegaron a Chile con diferentes propósitos y establecieron varios tipos de "encuentros culturales" -de corta duración, de larga data, problemáticos y amistosos- con los lugareños, siguiendo la tipología definida por Urs Bitterli (1989: 20-51). Estos encuentros eran esferas en las que tanto los británicos como los chilenos interactuaban, intercambiaban e influían mutuamente en sus respectivas culturas, expresadas en lenguaje, ideas, creencias, costumbres y actitudes. Esto encaja perfectamente en el análisis de Eliga H. Gould de las "historias entrelazadas" de Gran Bretaña y España, que están "relacionadas con la influencia mutua, las percepciones recíprocas o asimétricas y el proceso entrelazado de constituirse mutuamente" (Gould, 2007: 766). Estos intercambios tuvieron repercusiones significativas en la posterior configuración de una identidad nacional chilena, ya que fomentaron algunas de las primeras reflexiones y debates sobre el lugar que ocupaban los chilenos en el mundo. Los chilenos involucrados en las guerras de independencia, tanto en la marina como en el ejército, tuvieron que interactuar con individuos que hablaban otra lengua extranjera, profesaban otras religiones y tenían diferentes ideas sobre el comercio, la educación y la política.

La decisión de la Junta gubernativa, de abrir el comercio a naciones neutrales, motivó, por una parte, a algunas casas comerciales a enviar agentes con tal de explorar las primeras chances de establecer vínculos comerciales permanentes con Gran Bretaña. El caso más emblemático fue el de John James Barnard, quien arribó a Valparaíso en enero de 1812 proveniente de Valparaíso y que fue detenido por traficar armas en beneficio de los patriotas y violar el "pacto de naciones" en el que también se sustentaba la neutralidad británica. ${ }^{6}$ Por otro lado, Barnard operó como agente de la British and Foreign Bible Society y ya en 1813 había conseguido introducir ciento cincuenta co-

6 "Proceso contra Juan Diego Barnard", Santiago, noviembre de 1814, Archivo Nacional Histórico, Fondo Varios, vol. 440, f. 34. 
pias de la Biblia protestante en Chile. ${ }^{7}$ Con el tiempo, Barnard se convertiría en un mercader de cierta relevancia en la incipiente comunidad británica y que sería elegido como el secretario de la que pasaría a denominarse como la Association of British Merchants en 1817. La asociación se formó con el objetivo de facilitar las comunicaciones entre el almirante y un diverso grupo de mercaderes británicos instalados en Chile, transformándose en una vía para canalizar intereses particulares de sus asociados. Como señala John Rector, a través de su secretario la asociación tuvo una alta incidencia en la vida política de la década de 1820, especialmente en las formulaciones de la política económica (Rector, 1976: 21). Por otro lado, dicha asociación impulsó la aprobación de reformas para facilitar su incorporación en la sociedad chilena. Entre ellas, la aprobación de reformas a favor de una mayor tolerancia religiosa, puesto que, al ser en su mayoría protestantes, aspiraban a ejercer el derecho a enterrar a sus muertos en igualdad de condiciones con los católicos (Kinsbruner, 1970: 26-39).

Paralelamente a este proceso, la guerra de independencia continuó su curso. Aunque militarmente se produjeron triunfos trascendentes como el de Chacabuco (12 de febrero de 1817) y Maipú (1818), rondaba la convicción de que mientras no se expulsase a los españoles de los mares, la independencia jamás estaría asegurada, especialmente considerando la gran extensión costera del territorio chileno. Esto fue razón suficiente para que O'Higgins decidiera fundar la primera Escuadra de Chile el 20 de noviembre de 1817, comandada inicialmente por Manuel Blanco Encalada. A partir de noviembre de 1818 pasó a ser comandada por Lord Thomas Cochrane, lo que significó que junto a él se integrarían aproximadamente quinientos británicos, entre marinos y oficiales. El mismo Cochrane resumió su experiencia del siguiente modo:

Me veo obligado a informar o recordar de la peculiarmente ardua posición en la cual estuve como comandante de su escuadra, cuya tripulación estaba compuesta por un ensamblaje heterogéneo de nativos y extranjeros, sometidos a privaciones de las cuales no existe parangón en el servicio naval de los Estados europeos, ni siquiera en la marina mercante de algún país. ${ }^{8}$

En efecto, una de las características de la primera escuadra era la heterogeneidad de su tripulación, conformada principalmente por dos grandes (pero

7 "J. J. Barnard to Samuel Mills", 2 de agosto de 1813, Brtish and Foreign Bible Society Archive, Foreign Correspondence Inwards, Rev. J.J Barnard, Cambridge University Library. BSA $1 / 2$.

8 Thomas Cochrane, "Al Presidente y Gobierno de la República de Chile", 3 de febrero 1845, disponible en http://www.memoriachilena.cl/archivos2/pdfs/mc0034410.pdf. Revisada el 29-VI-2017. 
no exclusivos) grupos: quienes hablaban inglés y quienes hablaban español (Cubitt, 1976: 193-195). Entre medio estaban aquellos pocos que podían oficiar de traductores o intérpretes entre ambos y que fueron verdaderos mediadores culturales. Evidentemente, esto gatilló una serie de problemas de comunicación y conflictos al interior de la escuadra, incluso antes de la llegada de Cochrane. Varios oficiales británicos ya se habían enrolado en la escuadra y ocuparon la comandancia de algunas naves. Más bien por costumbre que como resultado de una medida específica, se estipuló que cada nave debía regirse por el idioma de su comandante (García Reyes, 1846. 10). Cochrane desconfiaba de la oficialidad chilena, por lo que a su llegada removió a todos los comandantes chilenos y los reemplazó por oficiales británicos o norteamericanos. De ese modo, en la práctica, la primera escuadra chilena se rigió oficialmente por el idioma inglés, al punto que un oficial de la Armada británica señaló que "incluso el uniforme es muy similar al nuestro". ${ }^{9}$

Una vez que Cochrane tomó el mando de la escuadra, surgió una nueva dificultad: qué regulación o norma utilizar en los barcos. Los británicos se regían por las Regulations y los chilenos por las Ordenanzas. ${ }^{10}$ Los británicos no estaban dispuestos a ceder en este punto, ya que desconocían la española y, además, se sentían portadores de una verdadera cultura marítima de la que los chilenos carecían (Kemp, 1970: 160-162). O'Higgins, no obstante, sabía que permitir esta nueva imposición podía ser problemático, dado que implicaría que todos los delitos y faltas se juzgarían según parámetros desconocidos para los chilenos. La solución en este caso fue diferente a la de los idiomas, pues el 16 de agosto de 1819 se estipuló que cada "individuo" sería regido por la normativa de acuerdo a su lugar de origen. ${ }^{11}$ Evidentemente, esto no evitó los problemas y, al contrario, generó una situación algo anómala: al ser la mayoría de los comandantes de origen británico, en la práctica imponían su normativa en el barco bajo su mando. Cualquier reclamo debía hacerse con posterioridad. Una situación conflictiva se vivió en la expedición hacia el Callao cuando Manuel Blanco Encalada exigió que en el barco que él viajaba se aplicase la normativa española, al ser el oficial de mayor rango. El comandante de la nave, William Wilkinson, se opuso tenazmente, afirmando que "las desconocía completamente", lo que generó una controversia que

9 "Thomas Hardy to John Wilson Croker", 17 de mayo de 1821. (Graham y Humphreys, 1952: p. 332).

10 Para los marinos británicos: Regulations and Instructions relating to his Majesty's Service at Sea. Esta es una versión revisada del King's Regulations and Admiralty Instructions, publicada en 1731. Para los marinos chilenos: Real ordenanza naval para el servicio de los baxeles de S.M. Es una versión revisada de las Ordenanzas Generales de la Armada Naval, publicadas en 1793 bajo el reinado de Carlos IV (1788-1808).

11 "O'Higgins al Senado", 16 de agosto de 1819, en Sesiones de los cuerpos lejislativos de la República de Chile, p. 161. 
finalmente fue solucionada por la mediación de Cochrane. ${ }^{12}$ Esto matiza en parte la idea planteada por Alamiro de Ávila Martel respecto a que la decisión de O'Higgins y del Senado de mantener diferentes normativas para cada grupo habría sido una nueva suerte de "fuero", similar al fuero colonial que permitía la existencia de grupos con diferentes privilegios a ojos del rey (Ávila, 1976: 135). Antes bien, podría decirse que las medidas decretadas fueron un intento por, al menos, preservar la identidad cultural de los chilenos y evitar una imposición excesiva de los parámetros culturales británicos.

Esto muestra, a diferencia de casos como el de Colombia, estudiados por Matthew Brown, que los conflictos no necesariamente se generaban en virtud del origen de unos u otros, o porque los chilenos visualizaran en los británicos a un nuevo imperio, sino que en virtud de condiciones de privación que eran comunes a todos. No obstante, es innegable que el hecho de que coexistieran diversos grupos, con diferentes idiomas, creencias y religiones fue un catalizador que llevó también a un sentido de "diferenciación" al interior de la escuadra, lo que redundaría también en una primera noción de que un grupo de individuos provenientes del territorio que acababa de independizarse tenía ciertas nociones y parámetros culturales comunes que debían reafirmar ante la presencia de un otro.

\section{El reconocimiento británico de la independencia de Chile}

Desde 1818 a 1831, identificamos una superposición en el rol tanto de actores estatales como no estatales, como resultado de la inauguración de las negociaciones para el reconocimiento británico de la independencia y la apertura del comercio, lo que alentó a miles de comerciantes a establecerse en Chile. Por otro lado, fue en este periodo también que arribaron misioneros protestantes británicos con la doble misión de difundir el sistema monitorial de enseñanza y circular biblias protestantes (Baeza, 2015 y 2016). En ese sentido, se aprecia la diversidad de intereses con la que diversos actores británicos arribaron a Chile, pues mientras unos venían representando al Estado y sus intereses comerciales, otros lo hicieron en virtud de sus propios intereses individuales o de otros actores privados como sociedades filantrópicas o casas comerciales. La consolidación de la independencia solo atrajo a más mercaderes y comerciantes, quienes comenzaron a establecerse en Valparaíso en dimensiones cada vez más crecientes, al punto que John Miers señaló que en 1822 ya había aproximadamente cuatrocientos británicos esta-

12 "Wilkinson a Cochrane", Archivo de Don Bernardo O'Higgins, 29 de octubre de 1819, doc. 225. 
blecidos en Valparaíso (Miers, 1826: 446). Dichos mercaderes gradualmente se incorporaron a la sociedad chilena y adquirieron un rol protagónico en las actividades económicas del periodo. Autores como Manuel Llorca-Jaña (2012) han destacado el hecho de que gracias a las actividades comerciales de estos mercaderes y de casas como Huth \& Co y Gibbs \& Co, Chile ingresó en una fase de globalización temprana, por cuanto las actividades de dichas casas eran también globales.

Una vez que se alcanzó y declaró la independencia de Chile (1818), las relaciones entre Gran Bretaña y Chile se mantuvieron también en un nivel "estatal". Esto se materializó, por un lado, en la iniciativa adoptada por el gobierno de O'Higgins en 1818 de enviar representantes del nuevo Estado a negociar el reconocimiento de su independencia a Londres. Para dicha misión fue comisionado Antonio José de Irisarri, en una misión con ciertas líneas de continuidad con la misión que había encabezado Pinto cinco años antes. La diferencia principal radicaba en que en esta oportunidad, Irisarri viajaba representando a un Estado cuya existencia se había declarado oficialmente el 12 de febrero de 1818. Sobre la necesidad del reconocimiento, se ha planteado desde la teoría jurídica que un Estado no llega a existir sino hasta que es reconocido por alguno ya existente. Esta visión, conocida como el modelo "constitutivista", se opone al modelo "declaratorista", que plantea que basta con que un Estado lleve a cabo una declaración formal de su independencia, o bien de su existencia, para que el resto de los Estados se vean en la obligación de reconocerlo (Grant, 1999: 4). En ese sentido, para determinar su incorporación al concierto de las naciones bastaría la sola declaración. En cierta forma, O’Higgins comprendió que ello no bastaba, especialmente por el contexto geopolítico en el cual las rivalidades inter-imperiales y la creciente hegemonía británica, sumado al rol que jugó la Santa Alianza, hacía necesario un reconocimiento por parte de dichos poderes. De hecho, fue Portugal el primer Estado en reconocer la independencia de Chile el 11 de agosto de 1821 y, luego, Estados Unidos el 22 de marzo de 1822.

No obstante, O'Higgins, al igual que sus sucesores, buscaría incesantemente el reconocimiento británico. Algunos autores han enfatizado que esto obedeció a la "anglofilia" de O'Higgins y su gran admiración por Gran Bretaña (Barros, 1970: 64; Racine, 2010: 434). Lo cierto es que considerando el contexto geopolítico y el creciente interés manifestado por Estados Unidos en extender sus operaciones hacia el Pacífico Sur, la opción de buscar un reconocimiento británico y la apertura de las relaciones diplomáticas formales, buscaba aminorar dicha amenaza y contar con un poderoso aliado que también pretendía extender sus circuitos comerciales hacia dicha zona. De hecho, fue a raíz de la exitosa operación de la Escuadra en la liberación de Lima, sumado al carácter altanero de Cochrane, lo que 
permitió configurar la idea de que Chile debía ser una potencia naval en el Pacífico, y también en el mundo. Por un lado, se planteó la posibilidad de que Chile emprendiera una expansión que abarcase hasta las Filipinas. De acuerdo a Luis Valencia Avaria, Cochrane habría discutido con O'Higgins esa posibilidad y en una carta le habría dicho: "conviértase usted en Emperador, Rey, Protector, Presidente o Jefe, o bajo cualquier título que le acomode mejor. Aquí está su Escuadra, que pondrá a sus pies cualquier cosa que existe en estas costas desde el Cabo de Hornos". O'Higgins, por su parte, habría respondido que una vez que se consolidara el dominio sobre Valdivia y Chiloé y se establecerían unas bases en Ecuador, "podríamos ir contra las Filipinas" (Valencia Avaria, 1942: 2-3). Por otro lado, en un documento posterior, O'Higgins se explayaría en su visión acerca de por qué Chile debía convertirse en una potencia naval mundial por medio de una alianza con Gran Bretaña y de ese modo debilitar los intereses de Estados Unidos (Silva Vildósola, 1923: 209-229).

Ahora bien, pese al entusiasmo mostrado por O'Higgins respecto a dicha alianza, las negociaciones para obtener el reconocimiento británico fueron interrumpidas y conflictivas. La misión de Irisarri fue un fracaso, pues no logró el reconocimiento, aunque logró conseguir el empréstito en Londres. De ese modo, fue reemplazado por Mariano Egaña en 1825, tras una decisión del gobierno de Ramón Freire. Egaña viajaría con nuevas instrucciones que reiteraban el objetivo de lograr el esquivo reconocimiento y especificaban nuevamente aquello que Egaña podía ofrecer a cambio. El principal problema para Egaña, no obstante, fue el nombramiento del primer cónsul británico en Valparaíso en octubre de 1823, Christopher Nugent. El creciente número de comerciantes y mercaderes británicos en Chile hizo necesario al nombramiento de una autoridad que velase por "los intereses comerciales de su Majestad británica" (Webster, 1938: 351-352). Sin embargo, fue precisamente la mala imagen difundida por Nugent sobre Chile lo que a juicio de Egaña impidió cualquier posibilidad de obtener el reconocimiento. Egaña reportó que "la opinión de que en Chile no hay orden, ni calma, ni orden gubernamental consolidado y tranquilo en Chile se fortalece" (González Echeñique, 1984: 123). En resumidas cuentas, los informes de Nugent daban cuenta de que Chile no era un Estado consolidado y mucho menos fiable. Dudaba de la integridad territorial y de la capacidad de Chile para defenderse ante una amenaza extranjera. En una de sus cartas reafirmó sus dudas acerca de la capacidad "tanto para invadir con alguna expectativa de éxito las posesiones de los realistas o incluso su capacidad, en el caso de un ataque desde Europa, de resistir" (Webster, 1938: 423). Esto era particularmente relevante debido a que Chiloé todavía permanecía bajo dominio español y Simón Bolívar ya comenzaba a amenazar a las autoridades chilenas que de no emprender una campaña para invadir e incorporar la isla a Chile, él mismo la invadiría desde el Perú (Gutiérrez Ardila, 2015). 
Esto también fue un aliciente para promover una nueva imagen sobre un país caracterizado por su orden, progreso y estabilidad y contrarrestar la mala fama difundida por Nugent. Fue en este proceso que los chilenos intentaron convencer a las autoridades británicas -infructuosamente- acerca de que Chile era un Estado cuya existencia podía estar asegurada y que no estaba sujeta a ningún otro poder. En otras palabras, la idea de Chile como un Estado libre y con cierta estabilidad política comenzó a ser discutida y defendida a propósito del anhelado reconocimiento británico de la independencia. Esta guerra de imágenes fue la que finalmente motivó a los chilenos a representarse a sí mismos como los ingleses pedían que fueran.

\section{Conclusiones}

Las relaciones entre chilenos y británicos durante el proceso de independencia distaron de ser unidireccionales y homogéneas. Adquirieron diversas facetas dependiendo del rol que asumía Gran Bretaña en el escenario de guerra que afectaba en ese entonces a Europa. Por ello, resulta sumamente importante considerar los cambios y giros experimentados en el periodo, debido a que eso determinó la forma en que los chilenos percibieron a Gran Bretaña. Un análisis cuidadoso en ese sentido sugiere que dichas percepciones cambiaron conforme el rol de Gran Bretaña cambiaba en el juego de alianzas inter-imperiales. Si en un primer momento la política británica hacia Chile fue de marcado carácter imperialista, al punto de amenazar con una invasión, luego se vio condicionada por la política de neutralidad frente al conflicto entre España y sus colonias. Eso abrió un nuevo escenario para actores "no estatales", que al emprender rumbo a Chile entraron en una dinámica de interrelaciones complejas y dinámicas que se experimentaron como contactos, colisiones o relaciones duraderas. Dichas interrelaciones resultaron fundamentales para la discusión de nociones como orden, estabilidad, progreso, que posteriormente formaron parte de la identidad cultural asociada a la nación chilena. Esta imagen se vio amenazada en el contexto de la negociación del reconocimiento británico, lo que llevó a los chilenos a defenderla y promoverla, a fin de lograr el tan anhelado reconocimiento.

\section{Referencias bibliográficas}

\section{Fuentes primarias}

a) Archivos y documentos

Archivo de Don Bernardo O'Higgins

Foreign Correspondence, British and Foreign School Society Archive, Uxbridge. 
Fondo Capitanía General, Archivo Nacional Histórico de Chile, Vol. 819.

Fondo Varios, Archivo Nacional Histórico de Chile, Vol. 237, 440.

War Office, National Archives at Kew, Londres.

\section{b) Impresos}

García Reyes, A. (1846). Memoria sobre la primera Escuadra nacional. Santiago: Imprenta del Progreso.

Letelier, V. (coord.) (1867). Sesiones de los cuerpos lejislativos de la República de Chile. Vol. 3 (1819-1820). Santiago: Imprenta Cervantes.

Real ordenanza naval para el servicio de los baxeles de S.M. (1802). Madrid: Imprenta Real.

Regulations and Instructions relating to his Majesty's Service at Sea (1808). London.

\section{Fuentes secundarias}

a) Artículos

Baeza, A. (2015). "Educational Reform, Political Change, and Penury: Primary Schooling and the Monitorial System of Education in Chile, approx. 1810-1833", en Marcelo Caruso (ed.). Classrom Struggle. Organizing Elementary School Teaching in the 19th Century, Studia Educationis Historica, Vol. 2. Frankfurt am Main: Peter Lang, pp. 67-90.

Baeza, A. (2016). "Circulación de biblias protestantes y tolerancia religiosa en la América post-independiente: La mirada de Luke Matthews, un misionero protestante británico, 1826-1829", en Economía y Política, Vol. 3, №2, pp. 5-35.

Baeza, A. (2017). "One Local Dimension of a Global Project: The Introduction of the Monitorial System of Education in Post-Independent Chile, 1821-1833", en Bulletin of Latin American Research, Vol. 36, № 3, pp. 340-353.

Blaufarb, R. (2007). "The Western Question: The Geopolitics of Latin American Independence", en The American Historical Review, Vol. 112, № 3, pp. 742-763.

Gallagher, J. y Ronald Robinson (1953). "The Imperialism of Free Trade", en The Economic History Review, Vol. 6, №1, pp. 1-15.

Gould, E. H. (2007). "Entangled Histories, Entangled Worlds: The English Speaking Atlantic as a Spanish Periphery", en The American Historical Review, Vol.112, N³, pp. 764-786.

Gutiérrez Ardila, D. (2017). "The Chilean Republic in the Face of Bolivar's Expansionism (1823-1828)", en Bulletin of Latin American Research, Vol. 36, $N^{\circ} 3$, pp. 313-325. 
Kinsbruner, J. (1970). "The Political Influence of the British Merchants Resident in Chile during the O'Higgins, administration, 1817-1823", en The Americas, Vol. 27, Nº1, pp. 26-39.

Lamperiere, A. (2013). "Presentación: Hacia una nueva historia transnacional de las independencias hispanoamericanas", en Jaime Rosenblitt (Ed.) Las revoluciones americanas y la formación de los estados nacionales. Santiago: DIBAM, pp. 13-27.

Ossa, J. L. (2010). "La Criollización de Un Ejército Periférico, Chile, 1768-1810", en Historia Vol. 43. Santiago, Instituto de Historia, Pontificia Universidad Católica de Chile, pp. 413-48.

Ossa, J. L. (2016). "1814 en Chile: de la desobediencia a Lima a la ruptura con España", en Anuario de Estudios Americanos, Vol. 73, №1, pp. 231-260.

Valencia Avaria, L. (1942). "Las Filipinas habrían podido ser para Chile", en En Viaje, Vol. 99, pp. 2-3.

Silva Vildósola, C. (1923). "Papeles de O'Higgins. Un proyecto de alianza chileno-británica", en Boletín de la Academia Chilena de la Historia, Vol. 17, pp. 209-229.

Waddell, D.A.G. (1987). "British Neutrality and Spanish-American Independence: The Problem of Foreign Enlistment", en Journal of Latin American Studies, Vol. $19 \mathrm{~N}^{\circ} 1$, pp. 1-18.

\section{b) Libros y tesis}

Amunátegui, M. (1876). La crónica de 1810, 3 vols. Santiago: Imprenta de la República de Jacinto Núñez.

Ávila Martel, A. (1976). Cochrane y la independencia del Pacífico. Santiago: Editorial Universitaria.

Bitterli, U. (1989). Cultures in Conflict. Encounters between European and NonEuropean Cultures, 1492-1800, Translated by Polity Press. Stanford, CA: Stanford University Press.

Barros, M. (1970). Historia diplomática de Chile, 1541-1938. Santiago: Andrés Bello.

Barros Arana, D. (1854). Historia jeneral de la Independencia de Chile, 2 vols. Santiago: Imprenta Chilena.

Brown, M. (2006). Adventuring Through Spanish Colonies. Simón Bolivar, Foreign Mercenaries and the Birth of New Nations. Liverpool: Liverpool University Press. 
Cubitt, D. (1974). "Lord Cochrane and the Chilean Navy, 1818-1823, with an inventory of the Dundonald papers relating to his service with the Chilean Navy" (tesis doctoral, inédita), 2 vols.

D'Arjuzon, Antoine (2003). Wellington. Madrid: Editorial Palabra.

Edmundson, W. (2009). A History of the British Presence in Chile. From Bloody Mary to Charles Darwin and the Decline of the British Influence. New York: Palgrave Macmillan.

Elliott, J. (2006). Empires of the Atlantic World: Britain and Spain in America, 1492-1830. New Haven: Yale University Press.

Gallo, K. (2001). Great Britain and Argentina. From Invasion to Recognition (1806-1826). Basingstoke and New York: Palgrave-St Antony's College, Oxford.

Guenther, L. H. (2004). British Merchants in Nineteenth-Century Brazil. Business, Culture, and Identity in Bahia, 1808-50. Oxford: Centre for Brazilian Studies, University of Oxford.

Graham, G. y R. A. Humphreys (eds.) (1952). The Navy in South America, 18071823. Correspondence of the Commanders-in-Chief on the South American Station. London and Colchester: Navy Records Society.

Llorca-Jaña, M. (2012). The British Textile Trade in South America in Nineteenth Century. Cambridge: Cambridge University Press.

Marshall, Peter (2005). The Making and Unmaking of Empires. Britain, India and America, c. 1750-1783. New York: Oxford University Press.

Mayo, J. (1981). "Britain and Chile, 1851-1886: Anatomy of a Relationship", en Journal of Inter-American Studies and World Affairs, Vol. 23, № 1, pp. 95-120.

Mayo, J. (1987). British Merchants and Chilean Development, 1851-1886. Boulder: Westview Press.

Montaner Bello, R. (1961). Historia diplomática de la independencia de Chile. Santiago: Editorial Andrés Bello.

Ossa, J. L. (2014). Armies, Politics and Revolution. Chile, 1808-1826. Liverpool: Liverpool University Press.

Rector, J. L. (1976). "Merchants, Trade, and Commercial Policy in Chile: 18101840", tesis doctoral inédita, Indiana University.

Robson, M. (2012). Britain, Portugal and South America in the Napoleonic War. London: I.B. Tauris.

Salazar, G. (2011). Mercaderes, empresarios y capitalistas: Chile, siglo XIX, 2nd ed. Santiago: Editorial Sudamericana. 
Terragno, R. (2001). Maitland y San Martín. Bernal: Universidad Nacional de Quilmes Ediciones.

Webster, Ch. K. (1938). Britain and the Independence of Latin America, 18121830, 2 vols. London, New York and Toronto: Oxford University Press. 